\title{
The development of a compact free spectral range semiconductor laser biosensor
}

J. Coote

j.coote@surrey.ac.uk

\section{S. Reddy}

\section{S. J. Sweeney}

Advanced Technology Institute, University of Surrey, Guildford, UK

Faculty of Health and Medical Sciences, University of Surrey, Guildford, UK

We present a new evanescent field sensor based on InP/InGaAsP ridge waveguide semiconductor lasers. The laser itself forms an integrated sensing chip containing both a light source and a waveguide. Simple modifications are made to readily available devices, meaning that the chips could form very compact and cost effective sensors. [DOI: 10.2971/je0s.2009.09013]

Keywords: biosensor, semiconductor laser, free spectral range

\section{INTRODUCTION}

When light propagates in a waveguide, the edges of the optical mode leak out from the waveguide core into the cladding and surrounding layers. Any change in the refractive index of the material this evanescent wave encounters will result in a change in the propagation constant of the mode, and therefore the effective refractive index of the waveguide. This makes waveguides with leaky evanescent fields highly suitable for biosensing applications, since a waveguide surface can be functionalised with biomolecules specific to the target molecule for sensing. When target molecules bind to the functionalised surface, this causes a change in the refractive index of the surface layer, thereby altering the effective index of the guided mode. The binding events can then be detected by monitoring certain characteristics of the light leaving the waveguide, which are dependent on the effective index.

A major advantage of this technique is that it does not require the use of fluorescent labels, as is the case with some other optical biosensor systems. Biosensing techniques such as surface plasmon resonance (SPR) [1] and dual polarisation interferometry [2] use the method of evanescent field sensing to make very powerful analytical tools, with high sensitivity and flexibility. However, these instruments make spatial measurements, such as SPR angle and the position of interference fringes, so need a physically large instrument (dimensions $\sim 10-100 \mathrm{~cm}$ ) to achieve high resolution.

Compact, portable and cost-effective sensors are needed for "in the field" and remote applications such as point of care medical diagnostics, environmental monitoring and security detection. Integrated optical sensing chips have the potential to meet these needs, and biophotonic devices based on tuneable semiconductor laser chips have been studied by Cohen and Coldren [3]. The approach we have taken is to use a stan- dard semiconductor laser and adapt it so that the laser itself becomes an integrated sensor chip.

In a conventional evanescent wave sensor, a light source illuminates a waveguide, the sample is in contact with the functionalised waveguide surface, and a detector records the output signal. A semiconductor laser chip contains both a light source, and a waveguide formed by the semiconductor layers which confine the laser light. In addition, semiconductor laser chips are very small with surface areas typically $\sim 0.1 \mathrm{~mm}^{2}$. We have therefore investigated using the laser itself as a highly compact integrated sensor, where the sample is brought into contact with the upper surface of the chip, and the emission spectrum is monitored for changes caused by the presence of the sample altering the effective index. Figure 1 shows a schematic diagram of the laser sensor.

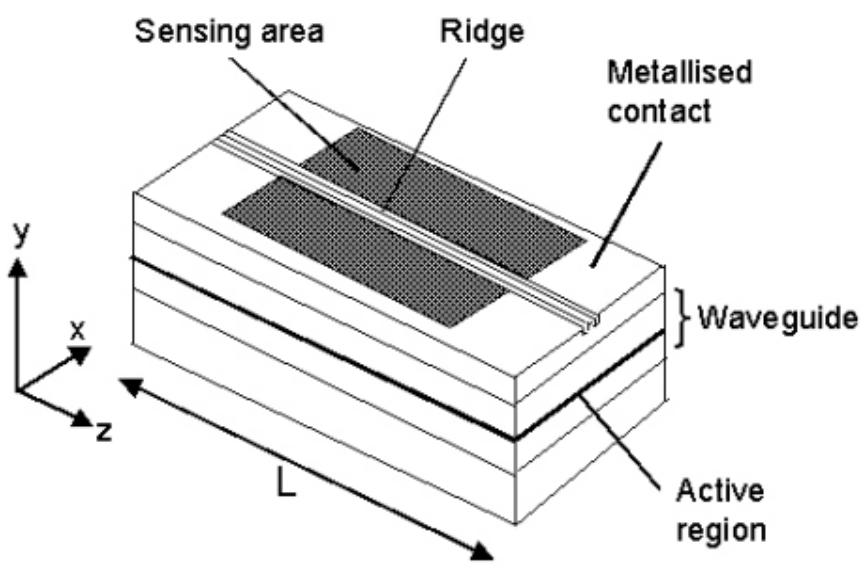

FIG. 1 Diagram of a ridge waveguide laser diode adapted for evanescent field sensing. The shaded regions on the surface of the chip are where the device has been milled to create a sensing area. 


\section{THEORY}

In an optimised laser structure, the evanescent field of the optical mode inside the semiconductor laser would not extend beyond the surface of the chip as this would lead to increased optical losses. For sensing applications, such losses are essential to the sensitivity hence surface modifications are required to achieve the desired sensing effect.

To investigate which modifications would be most beneficial, we created simplified 2-D models of laser waveguide structures and used a commercial mode solver (BeamPROP) to calculate the effective index of the guided modes when the refractive index of a thin surface test layer is varied. Details of this work are described in a previous paper [4]. Simulations showed that thinning the p-cladding layers above the laser active region greatly enhanced sensitivity by increasing the penetration depth of the evanescent field. However, this must be balanced against the need to maintain optical confinement in the active region as required for laser operation.

A typical Fabry-Perot laser spectrum contains a large number of peaks corresponding to modes of the laser cavity, The wavelength of the $m^{\text {th }}$ mode is given by

$$
\lambda_{m}=\frac{2 \mu_{e f f} L}{m}
$$

where $L$ is the length of the cavity and $\mu_{e f f}$ is the effective index. The free spectral range (FSR) is the spacing between Fabry-Perot modes in the laser spectrum, and is given by

$$
F S R=\frac{1}{2 \mu_{e f f} L}
$$

where FSR is expressed in wavenumbers. Eq. (2) is valid over wavelength ranges where the effective index varies only very slowly with wavelength and can be assumed constant. Changes in the effective index of the cavity can therefore be observed as shifts in both the free spectral range and the wavelength of individual modes. We define the sensitivity of the sensor device as the change in effective index with respect to the test layer index, or

$$
S=\frac{\partial \mu_{e f f}}{\partial \mu_{t}}
$$

where $\mu_{t}$ is the refractive index of the test layer. Therefore the change in FSR due to the change in test layer index can be written

$$
\Delta F S R=\frac{\partial F S R}{\partial \mu_{t}} \Delta \mu_{t}=\frac{\partial F S R}{\partial \mu_{e f f}} \frac{\partial \mu_{e f f}}{\partial \mu_{t}} \Delta \mu_{t}=-\frac{1}{2 \mu_{e f f}^{2} L} S \Delta \mu_{t}
$$

The relative magnitude of the shift in FSR is therefore

$$
\frac{\Delta F S R}{F S R}=\frac{1}{\mu_{e f f}} S \Delta \mu_{t}
$$

According to the modelling, a laser device with an optimised structure for this application would have a sensitivity of $1.97 \times 10^{-4}$ [4]. Taking $\mu_{\text {eff }}$ to be 3.52 (see Section 3), we can obtain a predicted shift in FSR for a change in $\mu_{t}$ of 0.4 :

$$
\frac{\Delta F S R}{F S R}=\frac{1.97 \times 10^{-4} \times 0.4}{3.52}=2 \times 10^{-5}=0.002 \%
$$

\section{EXPERIMENT}

A standard ridge waveguide laser with a wavelength of $1.55 \mu \mathrm{m}$ was milled using a focussed ion beam (FIB) to partially remove the gold contact and some of the uppermost device layer, creating two "windows" either side of the ridge. This created a sensing area on the surface of the chip. The positioning of the windows is depicted in Figure 1, and a SEM image of the milled surface of a laser device is shown in Figure 2 . The dimensions of the windows were $250 \mu \mathrm{m}$ long $\times$ $30 \mu \mathrm{m}$ wide $\times \sim 10 \mu \mathrm{m}$ deep. To simulate the effect of a biological sample binding to the sensor, a polymer film was deposited on to the surface of the chip, so that it covered the windows. The polymer used was cellulose acetate. Ellipsometry was used to determine the refractive index of a thin cellulose acetate film in the wavelength range 400-1000 nm, and the data were extrapolated using a Cauchy model to obtain a value of 1.4 for the refractive index of cellulose acetate at $1.59 \mu \mathrm{m}$. The average FSR of the laser over a wavelength range from $1.58 \mu \mathrm{m}$ to $1.6 \mu \mathrm{m}$ was measured. Over this range the effective index is approximately constant with respect to wavelength, and Eq. (2) is valid. Measurements of the FSR were taken before and after deposition of the polymer, using an optical spectrum analyzer with a resolution of $0.05 \mathrm{~nm}$, at a fixed injection current and base temperature. Repeat measurements were taken after removing the polymer layer with a solvent and then reapplying it.

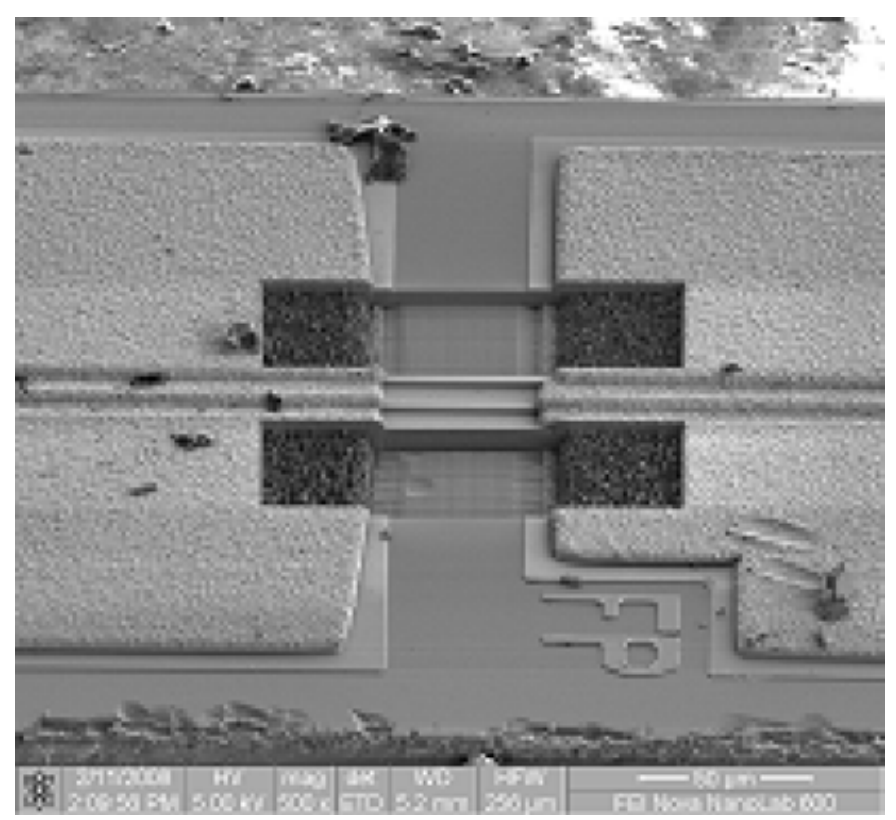

FIC. 2 SEM image of the surface of a milled laser.

Figure 3(a) shows part of the laser spectrum in reciprocal wavelengths, in one case where no sample was present (green line) and in the other where a layer of cellulose acetate covered the windows in the surface of the device. In Figure 3(b), a small section of the spectrum is enlarged to show individual Fabry-Perot modes. The free spectral range was extracted from the raw data by measuring the wavelength of two peaks at either end of the spectrum, at high resolution. The difference between these two wavelengths was divided by the number of Fabry-Perot modes (typically 75) between these two peaks to obtain an average value for the FSR. 

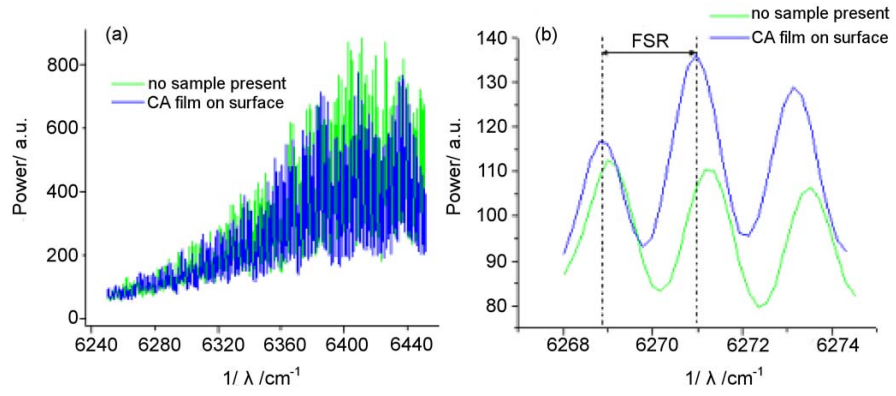

FIG. 3 (a) Spectra of the modified laser with no sample present (green line), and with a cellulose acetate film present (blue line). (b) Enlarged section of the spectrum, showing three Fabry-Perot modes.

As can be seen from Figure 3, the mode peaks shift to longer wavelengths when the polymer film is present, as would be expected if there had been an increase in the effective index of the cavity. There was also a reproducible negative shift in FSR when a polymer layer was applied to the device, and likewise a positive shift occurred when the layer was removed. Typically the shift in FSR between the case with no sample present and the case with a cellulose acetate sample present is around $0.02 \%$. From this result we can obtain an estimate for the sensitivity of the device, as defined in Eq. (3). Using Eqs. (4) and (5), the sensitivity $S$ can be written as

$$
S=\frac{\Delta F S R}{F S R} \frac{\mu_{e f f}}{\Delta \mu_{t}}
$$

An estimate of $\mu_{\text {eff }}$ can be obtained from the data by taking the initial value for the FSR, and the cavity length $L=650 \mu \mathrm{m}$, and using Eq. (2):

$$
\begin{gathered}
F S R=\frac{1}{2 \mu_{e f f} L}=2.187 \mathrm{~cm}^{-1} \\
\mu_{e f f} \approx \frac{1}{2 \times 2.187 \times 0.065}=3.52
\end{gathered}
$$

Using this value in Eq. (6), we obtain an estimate of the sensitivity $S$ :

$$
S \approx 0.0002 \times \frac{3.52}{0.45}=1.8 \times 10^{-3} .
$$

The observed shift in FSR of $0.02 \%$ and the estimated sensitivity of $1.8 \times 10^{-3}$ obtained from the experimental data is large compared to the predicted FSR shift and sensitivity for an optimised device of $0.002 \%$ and $1.97 \times 10^{-4}$ respectively. This is probably due to the fact that in the model a very thin test layer was used, whereas in these experiments the polymer layer is far thicker than the penetration depth of the evanescent field. In addition, the 2D modelling assumes the waveguide is uniform in the z-direction and completely covered with the film, whereas in the experiment the mode is only exposed to the film in a section of the cavity. This would result in a reduction in sensitivity compared to theory, but the effect would be small in comparison to the effect of the thick film.

Work is now in progress to characterise the sensor response by measuring the FSR shift with samples of different refractive indices and to expand the model to account for the effect of the window length relative to the laser cavity. The next step in the development of this laser biosensor would be to use distributed feedback lasers (DFBs). In a DFB laser, a Bragg grating provides feedback instead of the facets in a Fabry-Perot laser. The grating reflects only at a specific wavelength according to the Bragg condition, and this wavelength is strongly dependent on the effective index. Therefore with a DFB laser it should be possible to observe shifts in the peak emission wavelength as a measure of changes in surface index. The signals from two separate lasers, one acting as a sensor and the other as a reference, could be mixed and the beating frequency measured electrically, eliminating the need for a large piece of equipment such as a spectrometer. In addition, the use of two lasers would help to cancel noise caused by variations in current and base temperature, since if the two devices were mounted in close proximity and biased by the same current source, they should experience the same variations.

\section{CONCLUSIONS}

Semiconductor lasers have great potential in this new role as compact sensor chips. Simulations have shown that simple modifications to the laser structure can vastly improve sensitivity, and experimental results with standard lasers modified by FIB show promising results that confirm this. We predict that the sensitivity and compactness can be improved even further using a heterodyne technique with DFB lasers and optimised patterning.

\section{ACKNOWLED GEMENTS}

The authors gratefully acknowledge EPSRC for supporting JC. They also wish to thank Bookham for providing the lasers used in this study and Prof. Ben Murdin for helpful advice.

\section{References}

[1] U. Jonsson, L. Fagerstam, B. Ivarsson, B. Johnsson, R. Karlsson, K. Lundh, S. Lofas, B. Persson, H. Roos, I. Ronnberg, S. Sjolander, E. Stenberg, R. Stahlberg, C. Urbaniczky, H. Ostlin, and M. Malmqvist "Real-time biospecific interaction analysis using surface-plasmon resonance and a sensor chip technology" Biotechniques 11, 620-627 (1991).

[2] G. H. Cross, A. Reeves, S. Brand, M. J. Swann, L. L. Peel, N. J. Freeman, and J. R. Lu "The metrics of surface adsorbed small molecules on the Young's fringe dual-slab waveguide interferometer" J. Phys. D. Appl. Phys. 37, 74-80 (2004).

[3] D. A. Cohen, E. J. Skogen H. Marchand, and L. A. Coldren, “Monolithic chemical sensor using heterodyned sampled grating DBR lasers" Electron. Lett. 37, 1358-1360 (2001).

[4] J. Coote, S. Reddy, and S. J. Sweeney "Optimisation of distributed feedback laser biosensors" IET Optoelecton. 1, 266-271 (2007). 